Supporting Information

\title{
Photoactive Polyethylenimine-Coated Graphene Oxide Composites for Enhanced Cr(VI) Reduction and Recovery
}

\author{
Changwoo Kim, ${ }^{\mathrm{a}}$ Siyuan An, ${ }^{\mathrm{b}}$ Junseok Lee, ${ }^{\mathrm{a}}$ Anushree Ghosh, ${ }^{\mathrm{a}, \mathrm{b}}$ Mingjiang Zhong,,${ }^{\mathrm{a}, \mathrm{c}}$ \\ and John D. Fortner ${ }^{\mathrm{a}, *}$
}

${ }^{a}$ Department of Chemical and Environmental Engineering, Yale University, New Haven, CT 06520, United States

${ }^{\mathrm{b}}$ Department of Energy, Environmental, and Chemical Engineering, Washington University in St. Louis, St. Louis, MO, 63130, United States

${ }^{a}$ Department of Chemistry, Yale University, New Haven, CT 06520, United States

*To whom correspondence should be addressed:

John D. Fortner: Tel: +1-314-935-9293; Email: john.fortner@yale.edu 
(a)

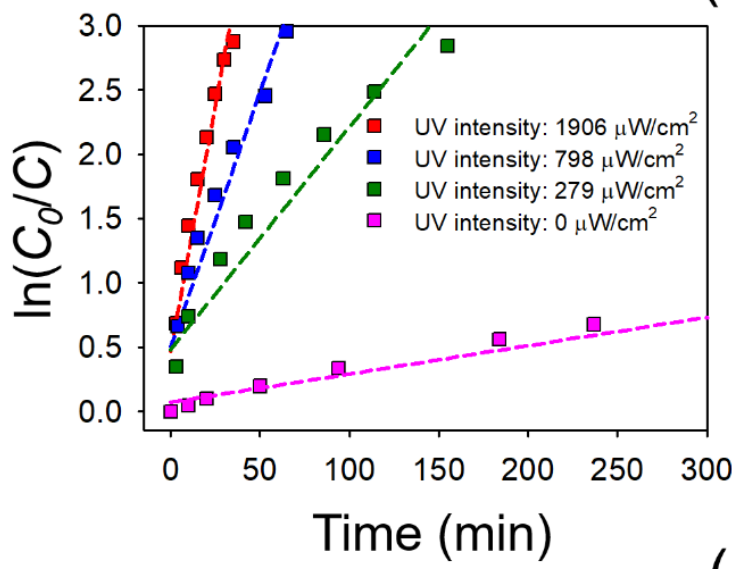

(b)

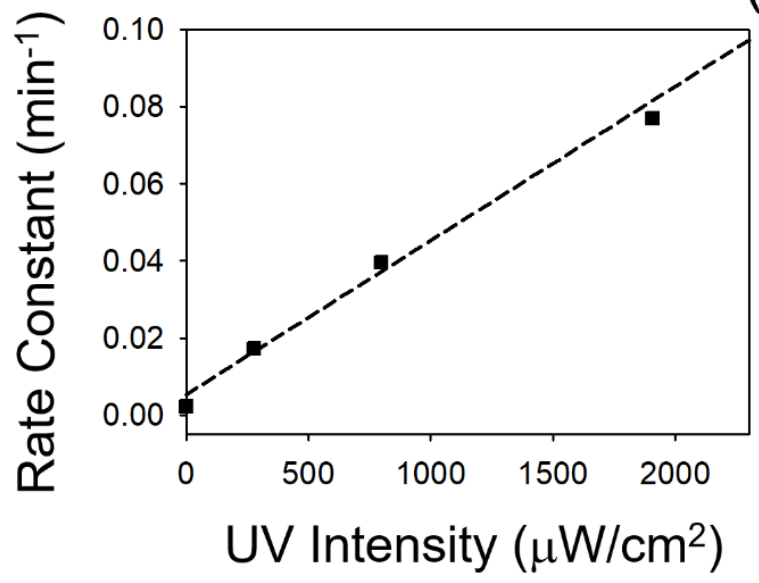

Figure $\mathrm{S} 1$ (a) Time dependent $\mathrm{Cr}(\mathrm{VI})$ removal as a function of UVA intensity $(\lambda=351 \mathrm{~nm})$. (b) Pseudo first order Cr(VI) reduction rate constants as a function UVA intensity. Pseudo first order $\mathrm{Cr}(\mathrm{VI})$ removal rate constants were $2.2 \times 10^{-3}, 1.7 \times 10^{-2}, 4.0 \times 10^{-2}$, and $7.7 \times$ $10^{-2} \mathrm{~min}^{-1}$ for light intensity of $0,279,798$, and $1906 \mu \mathrm{W} / \mathrm{cm}^{2}$, respectively. All experiments were conducted using $100 \mathrm{ppm}$ of PEI (branched, MW $25 \mathrm{~K}$ ) in the presence of $10 \mathrm{ppm}$ of $\mathrm{Cr}(\mathrm{VI})$ at $\mathrm{pH} 7 \pm 0.1$. 


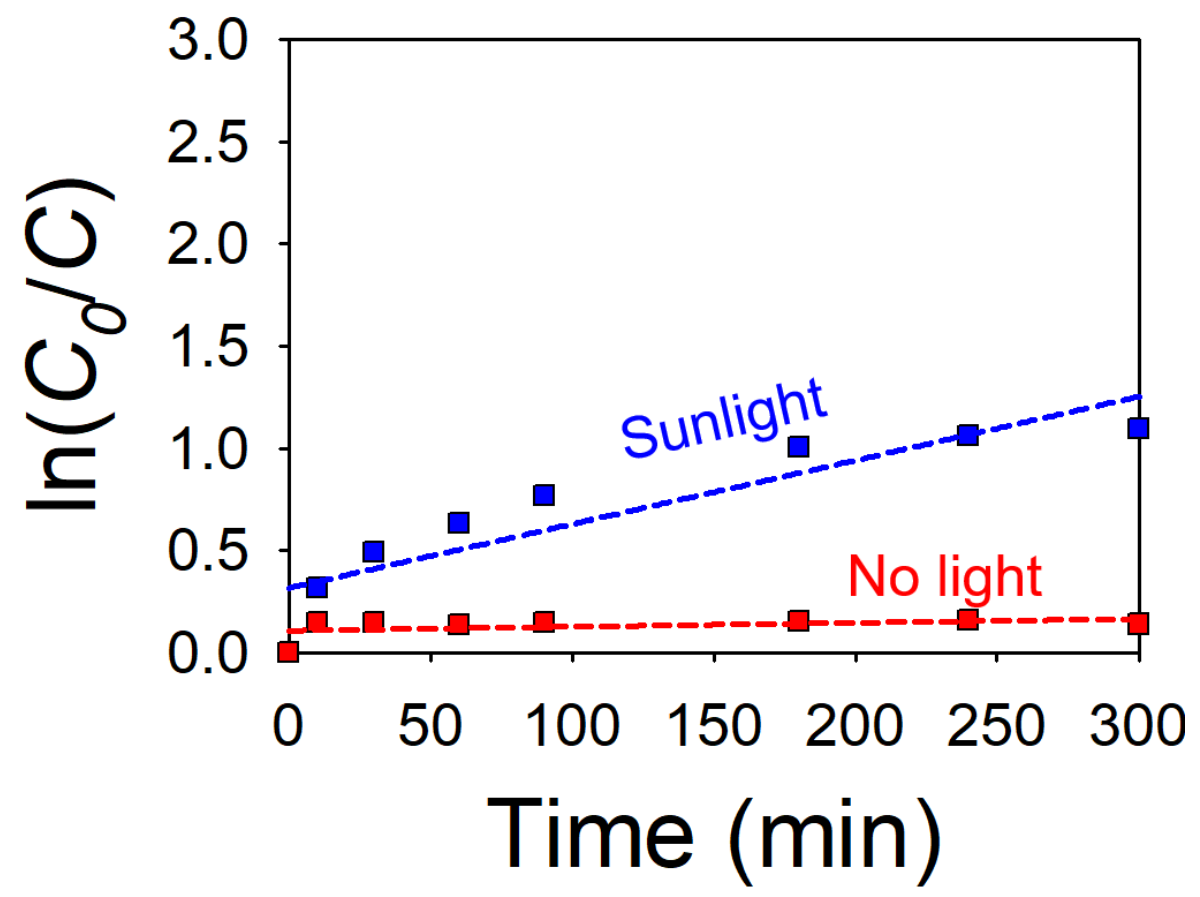

Figure S2 Time dependent $\mathrm{Cr}(\mathrm{VI})$ removal tests under real sunlight condition. The pseudo first order $\mathrm{Cr}(\mathrm{VI})$ removal rate constants were $3.1 \times 10^{-3}$ and $2.0 \times 10^{-4} \mathrm{~min}^{-1}$ for sunlight and no light condition, respectively. Every experiment was conducted using 100 ppm of PEI (branched, MW $25 \mathrm{~K}$ ) in the presence of $10 \mathrm{ppm}$ of $\mathrm{Cr}(\mathrm{VI})$ at $\mathrm{pH} 7 \pm 0.1$. Test date, time, sun exposure risk UV index, and temperature were November 6 2020, $11 \mathrm{AM}-4 \mathrm{PM}, 2$ of 10 (low), and $13-17^{\circ} \mathrm{C}$, respectively. 


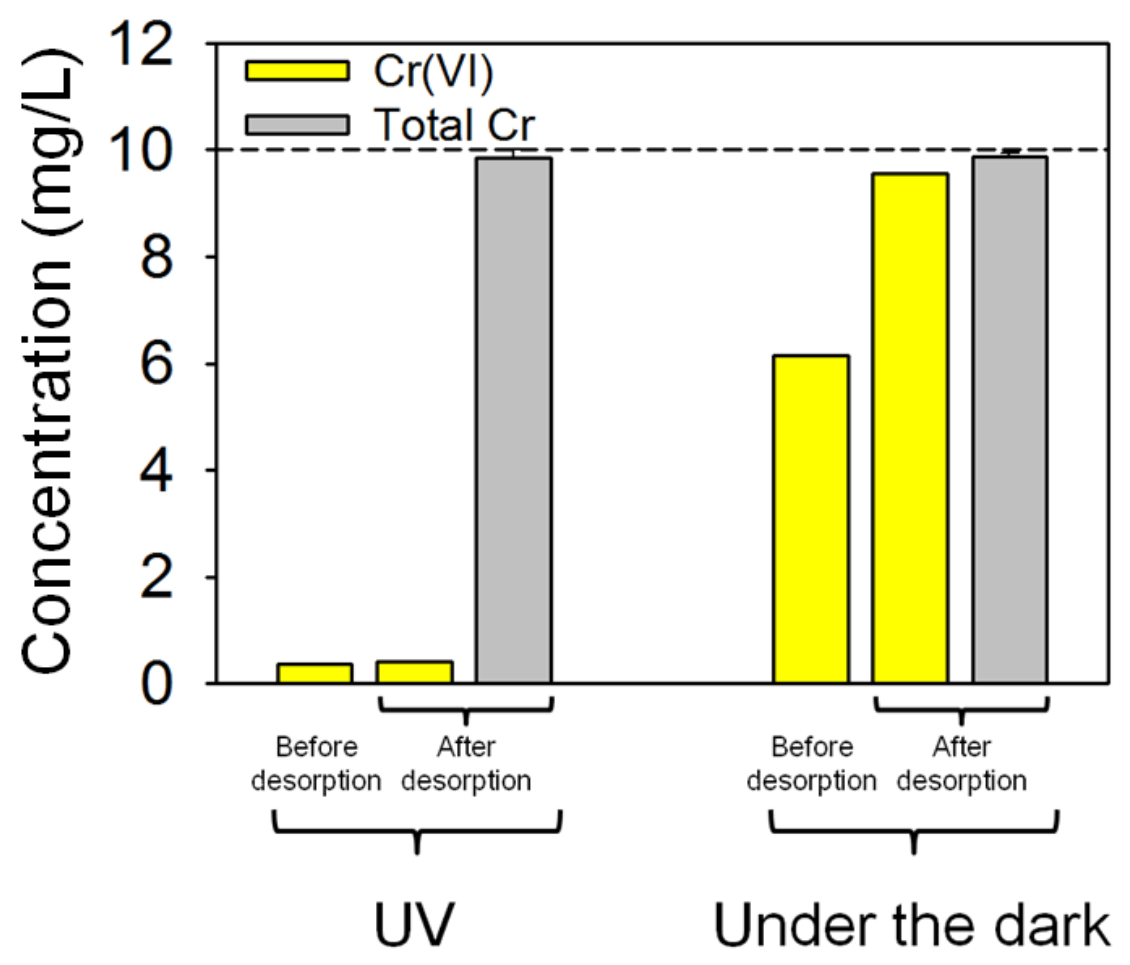

Figure S3 Total chromium and Cr(VI) concentrations before and after desorption. PEI samples were prepared using $100 \mathrm{ppm}$ of PEI (branched, MW $25 \mathrm{~K}$ ) in the presence of 10 ppm of $\mathrm{Cr}(\mathrm{VI})$ at $\mathrm{pH} 7 \pm 0.1$ for 200 min under the dark and UVA irradiation with two side lamp intensity $\left(950 \pm 50 \mu \mathrm{W} / \mathrm{cm}^{2}, \lambda=351 \mathrm{~nm}\right)$. 


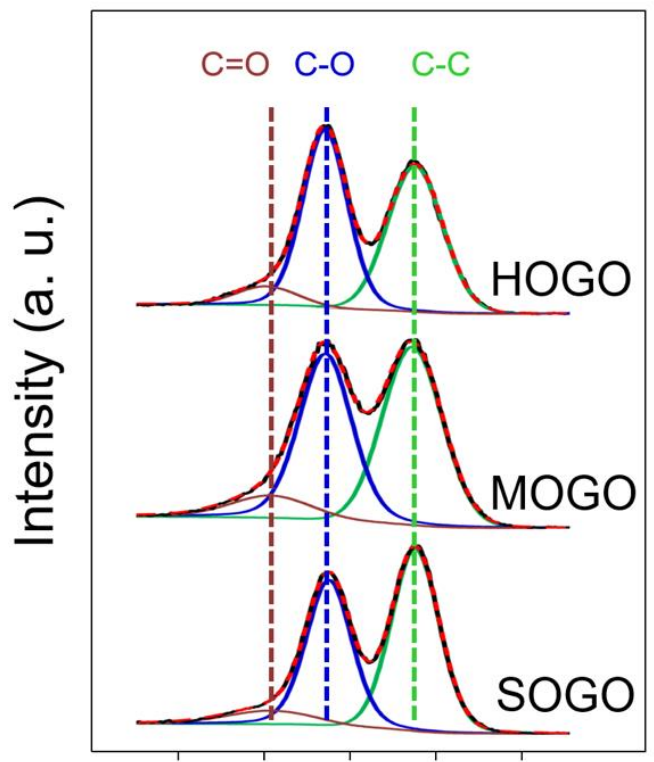

290288286284282

Binding Energy (eV)

Figure S4 The oxidation extent of GO was analyzed using XPS by measuring 1S carbon (C1S). The C1S spectra of SOGO, MOGO, and HOGO, deconvoluted into three types of carbon groups $\mathrm{C}-\mathrm{C}, \mathrm{C}-\mathrm{O}$, and $\mathrm{C}=\mathrm{O}$, whose binding energies were respectively $284.5,286.5$, and $287.8 \mathrm{eV}^{1}$ HOGO contained $45.9 \%, 47.6 \%$, and $6.6 \%$ of $\mathrm{C}-\mathrm{C}, \mathrm{C}-\mathrm{O}$, and $\mathrm{C}=\mathrm{O}$, respectively, MOGO had 48.2\%, 43.0\%, and $8.8 \%$ and SOGO possessed 51.3\%, 40.8\%, and $7.9 \%$. 


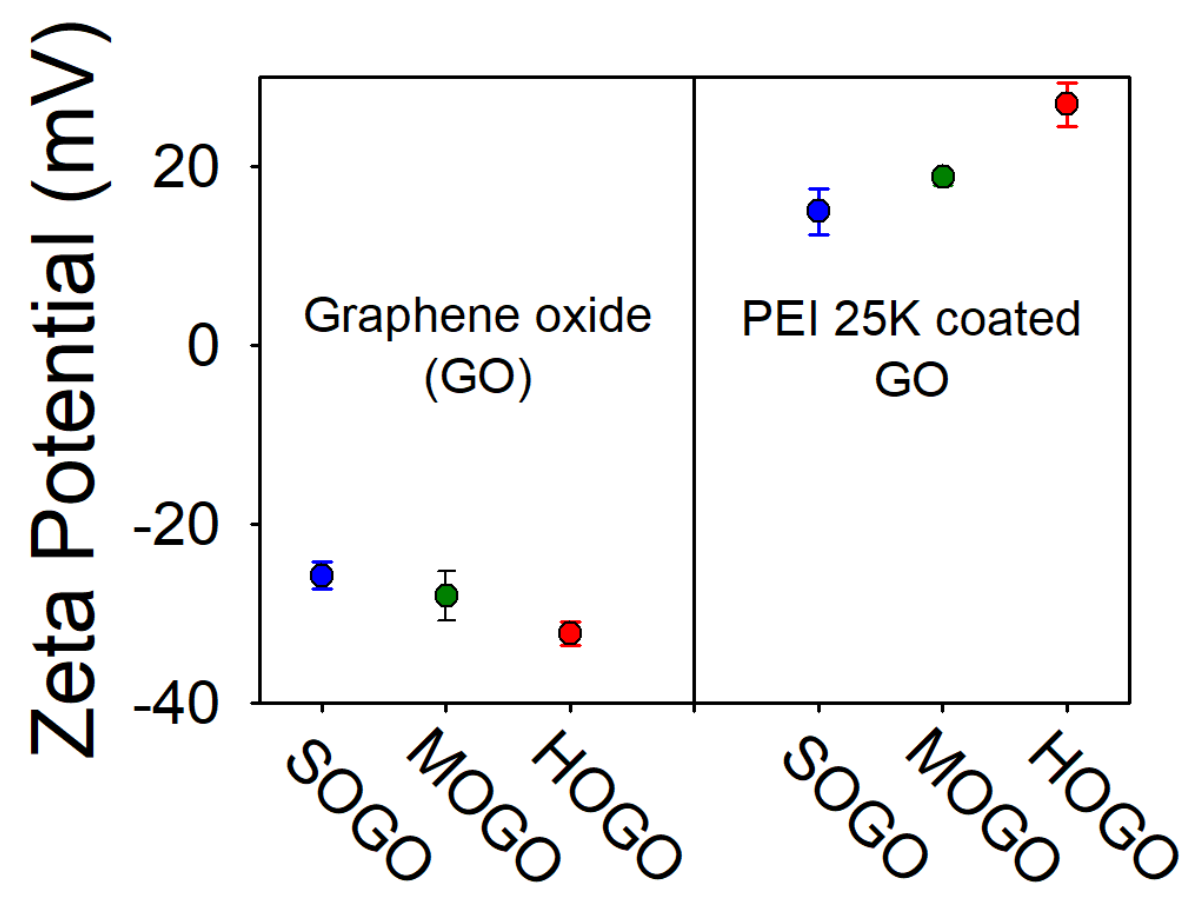

Figure S5 The zeta potentials of GO (SOGO, MOGO, and HOGO) and PEI coated GO (SOGO, MOGO, and HOGO) 


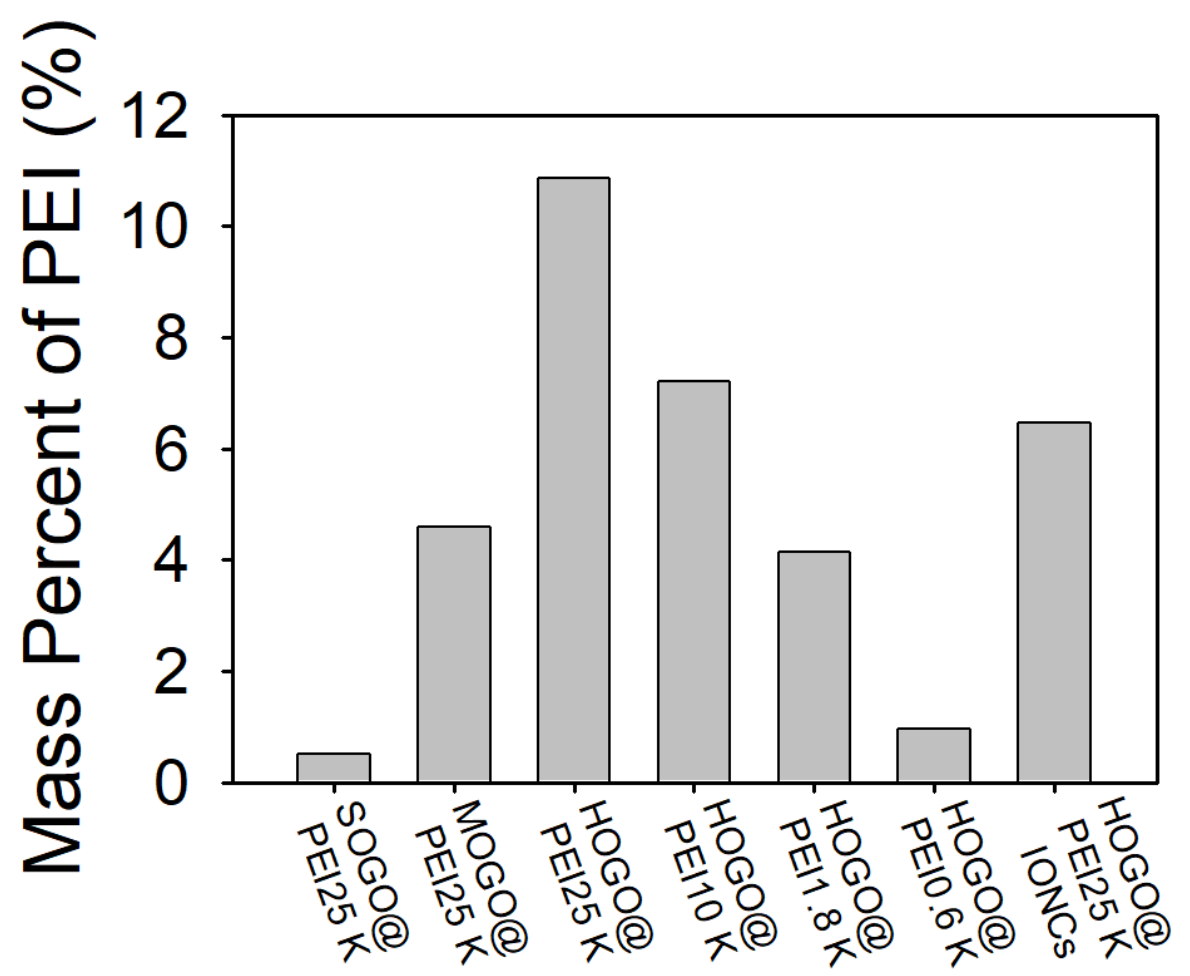

Figure S6 Mass percent of PEI for a series of GO@PEI and HOGO@PEI25 K@IONCs 


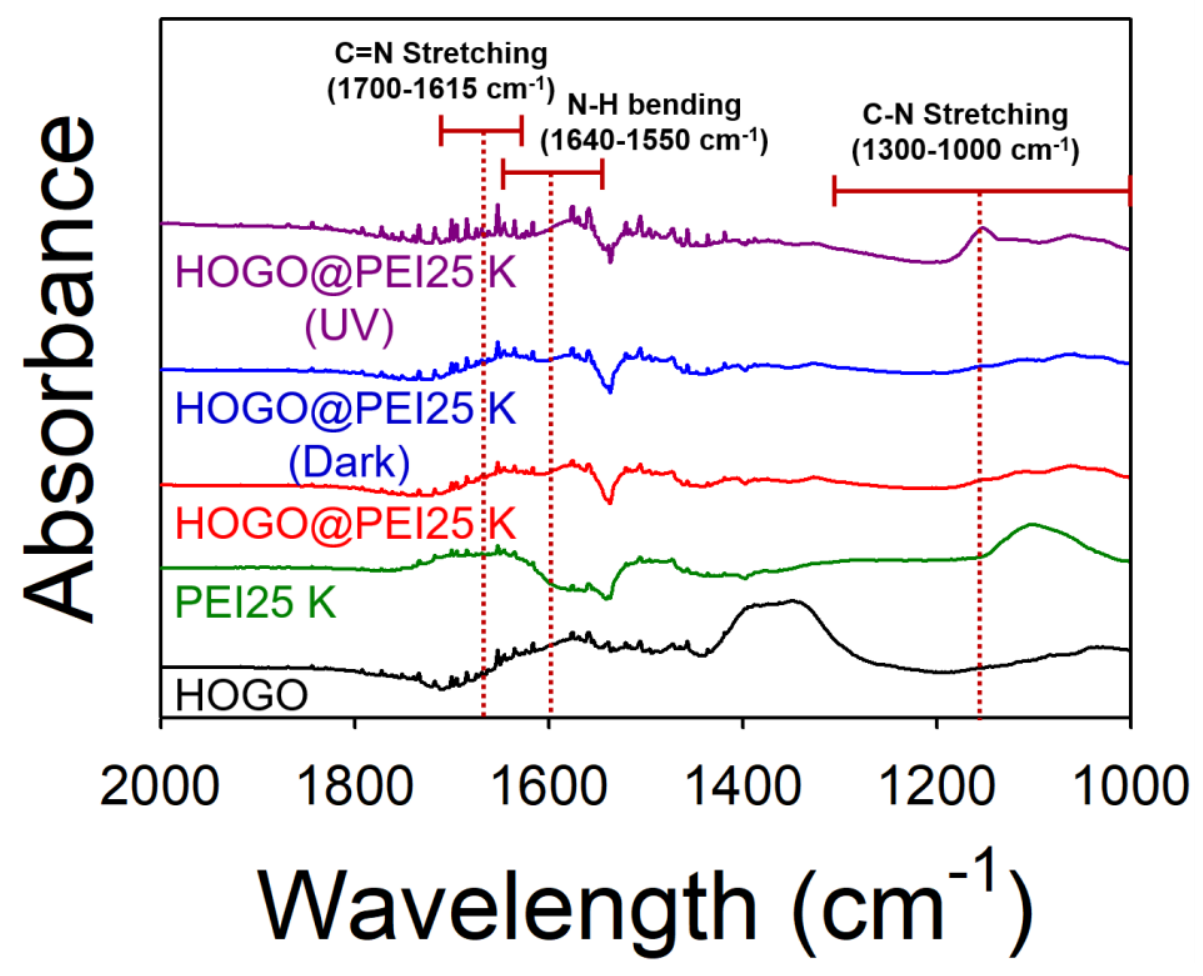

Figure S7 Fourier transform infrared spectroscopy (FTIR) measurements: HOGO@PEI25 K before and after experiment (in the dark and in UVA), PEI25 K, and HOGO 


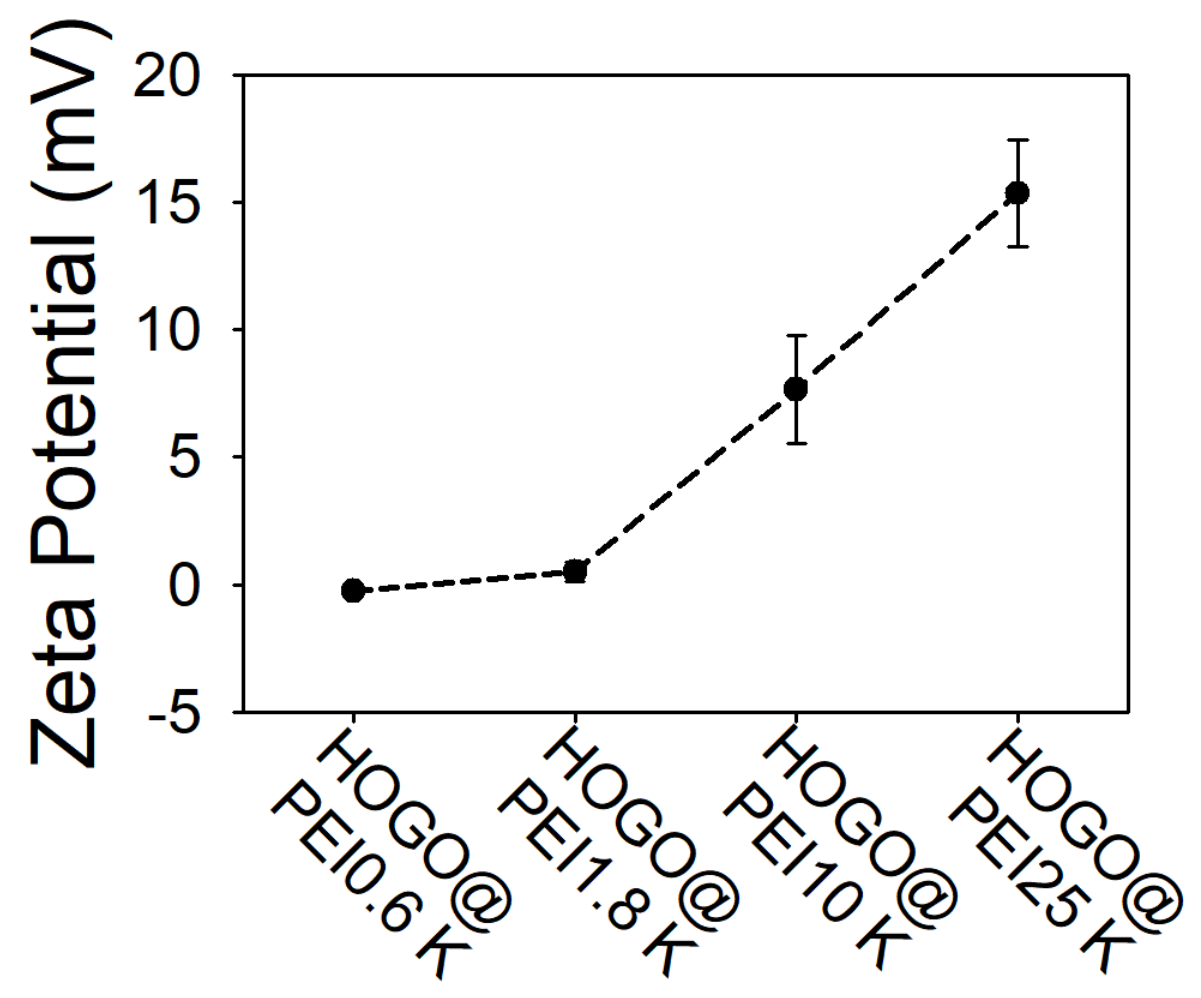

Figure S8 The zeta potentials of GO@ PEI as a function of MW of PEI 


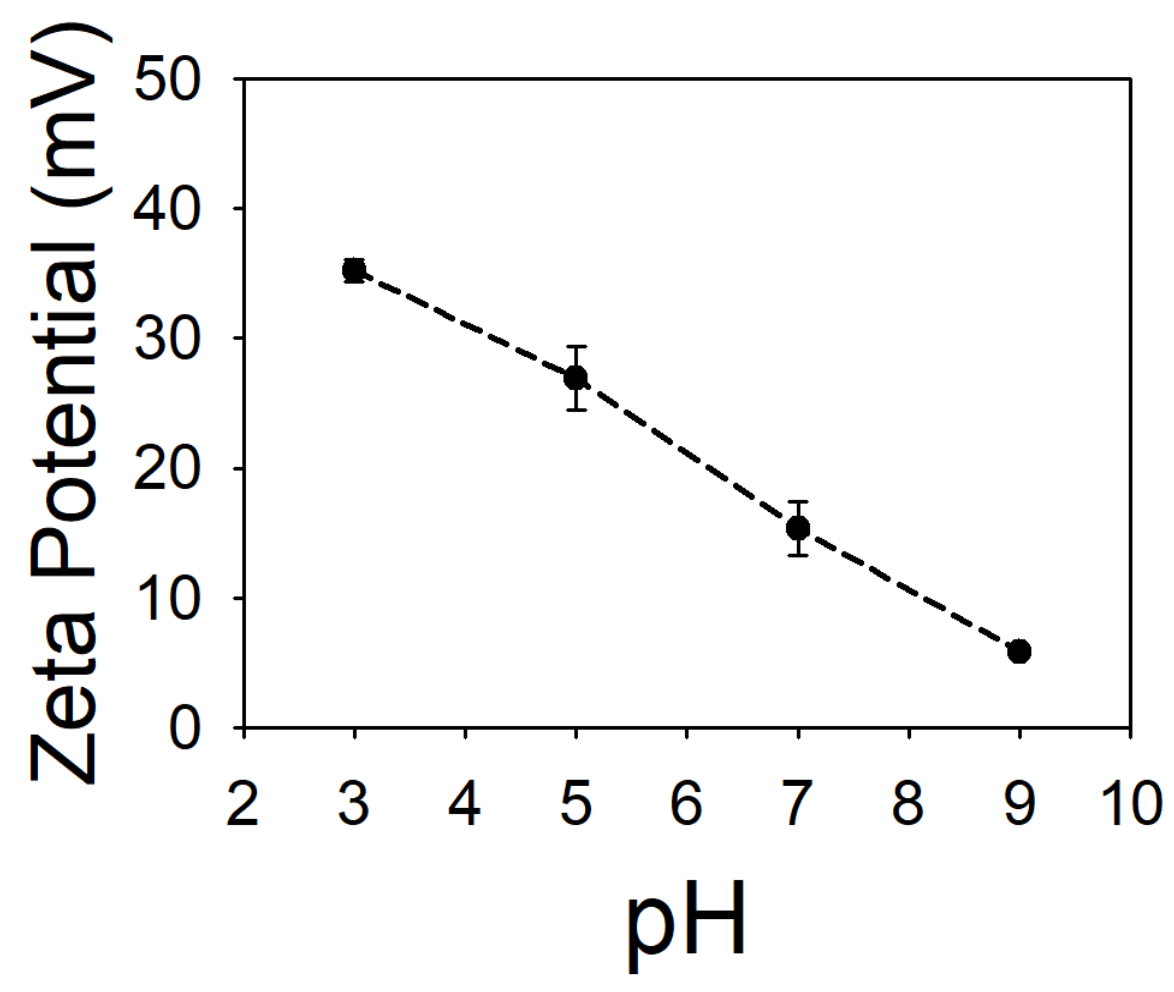

Figure S9 Zeta potential of HOGO@PEI25 K as a function of solution pH 


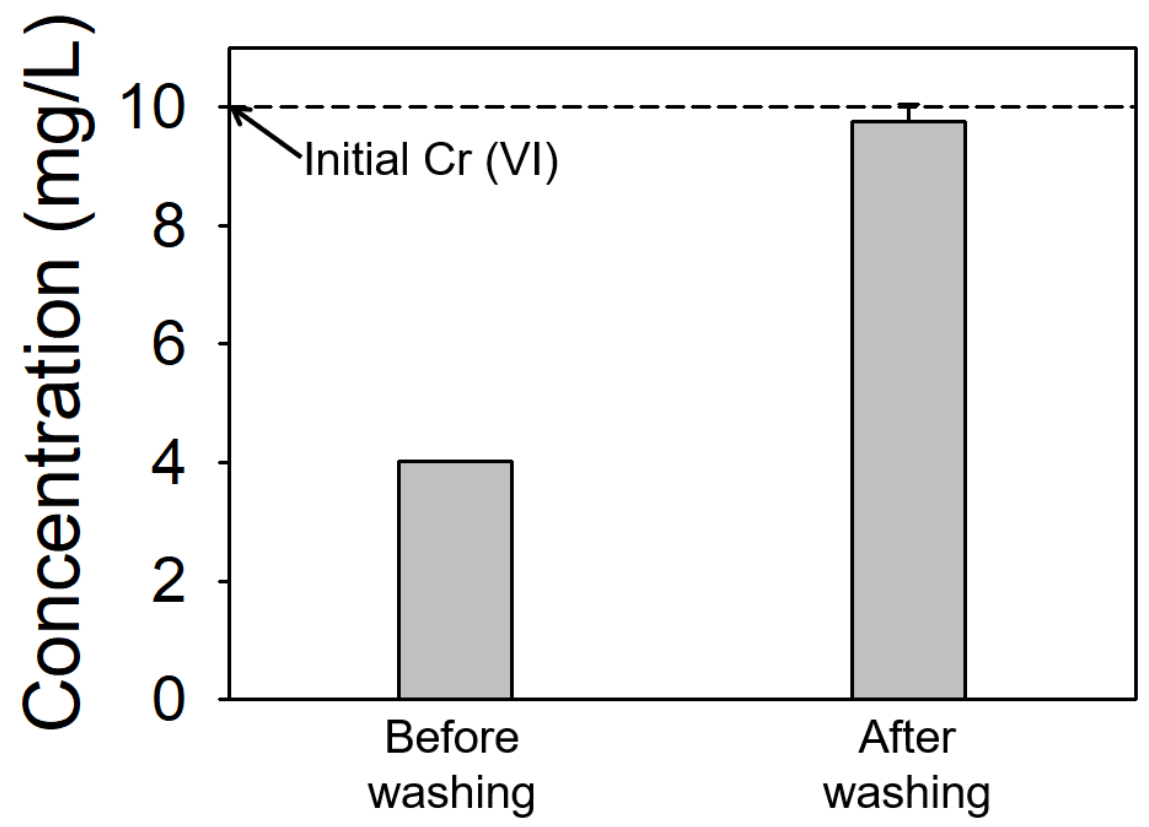

Figure S10 Cr(VI) concentrations before (pH 5) and after washing (pH 12) 
Table S1 List of organic compounds containing amine groups

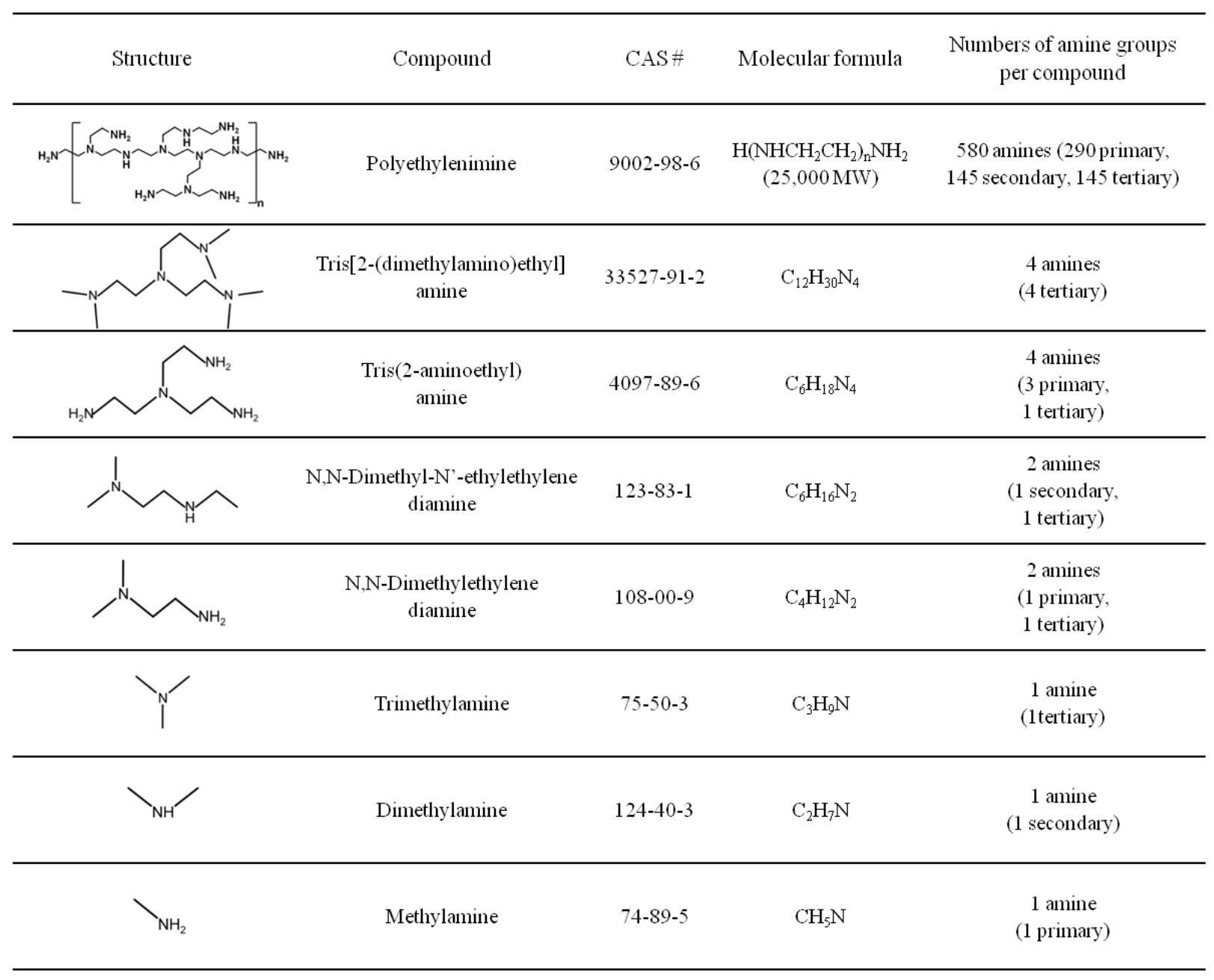


Table S2 Summary of adsorption capacities for $\mathrm{Cr}(\mathrm{VI})$ on amine functionalized materials

\begin{tabular}{|c|c|c|c|c|c|}
\hline Adsorbents & Organic compounds & Body composites & $\mathrm{pH}$ & $\begin{array}{l}\text { Isotherm } \\
\text { time }\end{array}$ & $\begin{array}{c}\mathrm{q}_{\mathrm{m}} \\
(\mathrm{mg} / \mathrm{g})\end{array}$ \\
\hline amino- $\mathrm{MA}^{2}$ & Amino groups & Mesoporous alumina & 2 & $3 \mathrm{~h}$ & 59.5 \\
\hline PANI-jute ${ }^{3}$ & Polyaniline & Jute fiber & 3 & $3-24 h$ & 62.9 \\
\hline MNP@PEI ${ }^{4}$ & Polyethylenimine & $\gamma-\mathrm{Fe}_{2} \mathrm{O}_{3} @ \mathrm{Fe}_{3} \mathrm{O}_{4}$ & 2 & $30 \mathrm{~min}$ & 78.1 \\
\hline Halloysite@polyaniline ${ }^{5}$ & Polyaniline & Halloysite clay nanotubes & 5 & $10 \mathrm{~min}$ & 82.6 \\
\hline 1D PANI ${ }^{6}$ & Polyaniline & Nanowire/tubes & 5 & $1 \mathrm{~h}$ & 86.8 \\
\hline sPANIs/Ch-HC ${ }^{7}$ & Polyaniline & Chitosan & 3 & $2 \mathrm{~h}$ & 147.2 \\
\hline PANI/HA ${ }^{8}$ & Polyaniline & Humic acid & 5 & $24 \mathrm{~h}$ & 150 \\
\hline Chitosan biosorbent $^{9}$ & Glucosamine & Chitosan & 4 & $24 \mathrm{~h}$ & 153.9 \\
\hline PEI-ESM ${ }^{10}$ & Polyethylenimine & Eggshell membrane & 3 & $24 \mathrm{~h}$ & 160 \\
\hline $\mathrm{PANI}-\mathrm{Fe} / \mathrm{OMC} \mathrm{C}^{11}$ & Polyaniline & $\begin{array}{l}\text { Magnetic mesoporous } \\
\text { carbon }\end{array}$ & 2 & $3 \mathrm{~h}$ & 172.3 \\
\hline PANI $^{12}$ & Polyaniline & - & 3 & $24 \mathrm{~h}$ & 198.7 \\
\hline $\mathrm{Fe}_{3} \mathrm{O}_{4} / \mathrm{PPy}^{13}$ & Polypyrrole & $\mathrm{Fe}_{3} \mathrm{O}_{4}$ & 2 & $24 \mathrm{~h}$ & 209.2 \\
\hline PP-nanofibers ${ }^{14}$ & Polypyrrole-polyaniline & Nanofibers & 2 & $24 \mathrm{~h}$ & 227.3 \\
\hline PANI/LDHs ${ }^{12}$ & Polyaniline & $\begin{array}{c}\mathrm{Mg} / \mathrm{Al} \text { layered double } \\
\text { hydroxides }\end{array}$ & 3 & $24 \mathrm{~h}$ & 393.7 \\
\hline Alginate@PEI ${ }^{15}$ & Polyethylenimine & Alginate & 2 & $24 \mathrm{~h}$ & 431.6 \\
\hline GO-2PEI ${ }^{16}$ & Polyethylenimine & Graphene oxide & 2 & $36 \mathrm{~h}$ & 436.2 \\
\hline PEI-PVA films ${ }^{17}$ & Polyethylenimine & Poly(vinyl alcohol) & 2 & $36 \mathrm{~h}$ & 396.8 \\
\hline PEI-USAH $^{18}$ & Polyethylenimine & Acidhydrochar & 2 & $6 \mathrm{~h}$ & 330.8 \\
\hline This study & Polyethylenimine & Graphene oxide & 3 & $5 \mathrm{~min}$ & 217.4 \\
\hline This study & Polyethylenimine & Graphene oxide & 5 & $5 \mathrm{~min}$ & 142.9 \\
\hline
\end{tabular}




\section{References}

(1) Dreyer, D. R.; Park, S.; Bielawski, C. W.; Ruoff, R. S. The Chemistry of Graphene Oxide. Chem. Soc. Rev. 2010, 39, 228-240.

(2) Cai, W.; Tan, L.; Yu, J.; Jaroniec, M.; Liu, X.; Cheng, B.; Verpoort, F. Synthesis of Aminofunctionalized Mesoporous Alumina with Enhanced Affinity Towards $\mathrm{Cr}(\mathrm{VI})$ and $\mathrm{CO}_{2}$. Chem. Eng. J. 2014, 239, 207-215.

(3) Kumar, P. A.; Chakraborty, S.; Ray, M. Removal and Recovery of Chromium from Wastewater Using Short Chain Polyaniline Synthesized on Jute Fiber. Chem. Eng. J. 2008, 141, 130-140.

(4) Pang, Y.; Zeng, G.; Tang, L.; Zhang, Y.; Liu, Y.; Lei, X.; Li, Z.; Zhang, J.; Liu, Z.; Xiong, Y. Preparation and Application of Stability Enhanced Magnetic Nanoparticles for Rapid Removal of $\mathrm{Cr}(\mathrm{VI})$. Chem. Eng. J. 2011, 175, 222-227.

(5) Zhou, T.; Li, C.; Jin, H.; Lian, Y.; Han, W. Effective Adsorption/Reduction of Cr(VI) Oxyanion by Halloysite@ Polyaniline Hybrid Nanotubes. ACS Appl. Mater. Interfaces 2017, 9, 6030-6043.

(6) Guo, X.; Fei, G. T.; Su, H.; De Zhang, L. High-Performance and Reproducible Polyaniline Nanowire/Tubes for Removal of Cr(VI) in Aqueous Solution. J. Phys. Chem. C 2011, 115, 16081613.

(7) Yavuz, A. G.; Dincturk-Atalay, E.; Uygun, A.; Gode, F.; Aslan, E. A Comparison Study of Adsorption of $\mathrm{Cr}(\mathrm{VI})$ from Aqueous Solutions onto Alkyl-Substituted Polyaniline/Chitosan Composites. Desalination 2011, 279, 325-331.

(8) Li, Q.; Sun, L.; Zhang, Y.; Qian, Y.; Zhai, J. Characteristics of Equilibrium, Kinetics Studies for Adsorption of $\mathrm{Hg}(\mathrm{II})$ and $\mathrm{Cr}(\mathrm{VI})$ by Polyaniline/Humic Acid Composite. Desalination 2011, 266, 188-194.

(9) Boddu, V. M.; Abburi, K.; Talbott, J. L.; Smith, E. D. Removal of Hexavalent Chromium from Wastewater Using A New Composite Chitosan Biosorbent. Environ. Sci. Technol. 2003, 37, 4449-4456.

(10) Liu, B.; Huang, Y. Polyethyleneimine Modified Eggshell Membrane as A Novel Biosorbent for Adsorption and Detoxification of $\mathrm{Cr}(\mathrm{VI})$ from Water. J. Mater. Chem. 2011, 21, 1741317418.

(11) Yang, G.; Tang, L.; Cai, Y.; Zeng, G.; Guo, P.; Chen, G.; Zhou, Y.; Tang, J.; Chen, J.; Xiong, W. Effective Removal of Cr(VI) Through Adsorption and Reduction by Magnetic Mesoporous Carbon Incorporated with Polyaniline. RSC Advances 2014, 4, 58362-58371. (12) Zhu, K.; Gao, Y.; Tan, X.; Chen, C. Polyaniline-Modified Mg/Al Layered Double Hydroxide Composites and Their Application in Efficient Removal of Cr(VI). ACS Sustain. Chem. Eng. 2016, 4, 4361-4369.

(13) Wang, Y.; Zou, B.; Gao, T.; Wu, X.; Lou, S.; Zhou, S. Synthesis of Orange-Like $\mathrm{Fe}_{3} \mathrm{O}_{4} / \mathrm{PPy}$ Composite Microspheres and Their Excellent Cr(VI) Ion Removal Properties. J. Mater. Chem. 2012, 22, 9034-9040.

(14) Bhaumik, M.; Maity, A.; Srinivasu, V.; Onyango, M. S. Removal of Hexavalent Chromium from Aqueous Solution Using Polypyrrole-Polyaniline Nanofibers. Chem. Eng. J. 2012, 181, 323-333.

(15) Yan, Y.; An, Q.; Xiao, Z.; Zheng, W.; Zhai, S. Flexible Core-Shell/Bead-Like Alginate@ PEI with Exceptional Adsorption Capacity, Recycling Performance Toward Batch and Column Sorption of Cr(VI). Chem. Eng. J. 2017, 313, 475-486. 
(16) Geng, J.; Yin, Y.; Liang, Q.; Zhu, Z.; Luo, H. Polyethyleneimine Cross-Linked Graphene Oxide for Removing Hazardous Hexavalent Chromium: Adsorption Performance and Mechanism. Chem. Eng. J. 2019, 361, 1497-1510.

(17) Liu, W.; Wang, M.; Wen, Z.; Li, Z.; Yang, L.; Jiang, C. Recyclable Functionalized Polymer Films for the Efficient Removal of Hexavalent Chromium from Aqueous Solutions. RSC Advances 2019, 9, 36751-36757.

(18) Wang, J.; Xie, Q.; Li, A.; Liu, X.; Yu, F.; Ji, J. Biosorption of Hexavalent Chromium from Aqueous Solution by PEI-Modified Ultrasonic Assisted Acidhydrochar from Sargassum Horneri. Water Sci. Technol. 2020, 81, 1114-1129. 